\title{
Influences of Levels of Processing, Misleading Intensity and Warnings on Memory Suggestibility
}

\author{
Xiao-Jun Cao ${ }^{1 *}$, Shen Tu${ }^{1}$, Xu Chen ${ }^{2 *}$ \\ ${ }^{1}$ Institute of Education, China West Normal University, Nanchong, China \\ ${ }^{2}$ Faculty of Psychology, Southwest University, Chongqing, China \\ Email: cxj841118@aliyun.com, ${ }^{*}$ chenxu@swu.edu.cn
}

Received 2 April 2015; accepted 7 May 2015; published 8 May 2015

Copyright (C) 2015 by authors and Scientific Research Publishing Inc.

This work is licensed under the Creative Commons Attribution International License (CC BY).

http://creativecommons.org/licenses/by/4.0/

(c) (i) Open Access

\section{Abstract}

The influences of levels of processing at the encoding phase, misleading intensity at the misleading phase and warning at the retrieval phase on memory suggestibility were explored through the delayed retrieval errors paradigm, and the contributions of dynamic automatic processing and intentional processing to generation of memory suggestibility were deeply assessed through the Remember/Know/Guess paradigm. The results showed that levels of processing were an important influencing factor for memory suggestibility. The intentional processing at the encoding phase benefited the generation of memory suggestibility more than the automatic processing. The misleading intensity had a significant effect, where the misled-generate items were recalled at a higher level with a longer response time than the misled-read items. In addition, the warning increased both the memory suggestibility of misleading pictures and the memory accuracy of non-misleading pictures. This memory suggestibility was jointly caused by the automatic processing and intentional processing, but mainly intentional processing, and the warning increased the probability of intentional processing during the generation of memory suggestibility.

\section{Keywords}

Memory Suggestibility, Levels of Processing, Warnings, Automatic Processing, Intentional Processing

\section{Introduction}

There are two types of false memories: associative false memories and misleading false memories. Misleading

${ }^{*}$ Corresponding authors. 
false memories can be interpreted as the individual's susceptibility to misleading information, i.e. memory suggestibility. In a broad sense, memory suggestibility means that the encoding, maintenance, retrieval and reporting of an event are influenced by a social and psychological factor (Zhou \& Liu, 2003). Numerous studies had explored this effect at both encoding and retrieval of associative false memories (McDermott \& Watson, 2001; Dewhurst et al., 2009; Arndt, 2006), and uniformly concluded that the false memories were jointly generated by both encoding and retrieval processing. Therefore, memory suggestibility is influenced directly by variables during both encoding and retrieval.

At present, the influence of levels of processing at the encoding phase on false memories is still inconsistent. The following types of manipulations do not influence false memories: pleasure evaluation, letter counting, and judgment of initial vowel in a word (Tussing \& Greene, 1997). Deep levels of processing leads to higher incidence rates of inaccurate recall as well as higher rates of accurate recall, indicating that inaccurate recall is influenced by levels of processing (Rhodes \& Anastasi, 2000). Ye \& Liu (2008) found that the associative intensity and levels of processing were the important influencing factors for generation of false memories through DRM (Deese-Roediger-McDermott) paradigm, i.e. under a strong relational sequence, deep levels of processing led to higher rates of inaccurate recognition than shallow levels of processing, but under a weak relational sequence, shallow levels of processing led to higher rates of inaccurate recognition than deep levels of processing. Related to these findings, the effect of levels of processing on memory suggestibility requires the further study.

A dominant viewpoint is that warnings can effectively reduce false memories (Gallo et al., 1997, 2001). Zhou \& Nie (2009) found that the warnings at the encoding phase could lead to a strict judgment criterion for recognition, so as to reduce effects of false memories. But from Li, Lian, \& Weng (2008), using the signal detection analysis, a warning did not significantly influence false memories (Westerberg \& Marsler, 2006). Peters et al. (2008) demonstrated that accurate memory was reduced and that the embedding memory of key lures was increased by reducing the attention level during the encoding, which could not be avoided even through the warning words. When attentional control and warnings have to compete for limited cognition resources during free recall, the attentional distraction can increase the inaccurate recall. Therefore, warning words cannot fully inhibit false memories induced under the test conditions. In the misinformation interference paradigm, although all subjects were warned of false memories, some subjects were still interfered by misinformation to produce false memories (Guo et al., 2005). In a word, a consensus is not yet reached on what influence of warnings on false memories. In most past studies, subjects were warned of appearance of misinformation, so as to reduce the false memories. Here, the question becomes, "do the same results occur when subjects are warned after the appearance of misinformation but before the start of recognition test?" The effectiveness of warning words might be related to the types of encoding information and the retrieval fluency of warning information at the test phase. Most studies on warnings of false memories were inclined to explore the effects at the learning phase, but did not pay attention to the effects at the retrieval phase. Providing that warnings increase the monitoring level of subjects at the retrieval phase, what relationship does such increase have with the generation of memory suggestibility? All these key questions would be answered in our study.

Memory suggestibility is processed in a unique way, i.e. a misleading phase is added between encoding phase (learning phase) and retrieval phase (test phase). Yang, Wang, \& Tang (2006) found that associative false memories might occur at the encoding phase of memories, but misleading false memories were more likely to occur at the misleading phase. Therefore, in the studies on the influence mechanism of memory suggestibility, a close attention should be paid to not only the relevant variables at the routine encoding/retrieval phase, but also the effects of influencing factors at the misleading phase (i.e. a unique processing phase of memory suggestibility).

As indicated by Fuzzy-trace theory of Brainerd \& Reyna (1998), the afterward misinformation was reported by subjects not only through intentional processing but also through automatic processing. If an inaccurate recognition is jointly produced by the automatic processing and intentional processing, it is very important to determine the contribution of each processing to memory suggestibility. By integrating levels of processing at the encoding phase, misleading intensity at the misleading phase, and warnings at the retrieval phase, our study explored the single effect and complex interaction of important influencing factors at these phases, and further clarified the contributions of automatic processing and intentional processing during the generation of memory suggestibility. The results of our study would reveal the mechanism of memory suggestibility and play a certain role of theoretical guidance in rationally utilizing the hinting principles in the educational practice. 


\section{Methods}

\subsection{Subjects}

In the Southwest University (Chongqing, China), 72 undergraduate volunteers were recruited for our study. 68 valid subjects were randomly divided into two groups: non-warning group (19 males and 15 females) and warning group (17 males and 17 females). The subjects were at an age range of $18-25$ years old, dextromanuality, and normal vision or corrected vision. After the completion of test, all subjects were paid at a certain amount.

\subsection{Stimulus Materials}

At the formal test, a figured story of about 1000 words was selected, and the fluency and comprehensibility of its sentences were corrected and polished by two postgraduates at the Chinese Language Department. At the learning phase, the story words were presented to subjects sentence by sentence, and then 51 pictures corresponding to details of the sentences were presented (each picture represented one independent thing). At the misleading phase, a misleading literal summary of about 800 words was compiled according to the lines and details of the story presented at the learning phase. In this literal summary, there were 48 details for memories, including 24 control items (which were the same as the original story), 12 misled-read items (which changed details of the story) and 12 misled-generate items (at the same time of changing the story details, the order of words was upset on purpose, and then subjects were asked to re-arrange it into accurate order, which aimed to increase the conscious processing of misleading items, i.e. increase the misleading intensity). At the recognition test phase, 48 pictures of 4 types were presented to subjects, including 12 old pictures at the learning phase, 24 misleading pictures corresponding to misleading literal summary at the misleading phase (including 12 misled-read pictures and 12 misled-generate pictures), and 12 new pictures not ever presented or mentioned. All story pictures were chosen from web photo gallery, and were jointly selected by two doctors of psychology (i.e. experimenters). Each picture was evaluated in terms of valence, content familiarity and identification complexity. According to the scores of two experimenters, these pictures were classified into 4 types, and each type of pictures was matched in terms of valence, content familiarity and identification complexity. The pictures were colored, mainly for the expressions, activity and apparel of persons, and the characteristics of relevant things. The pictures were in a resolution of 72 elements/inch (size $15.23 * 10.98 \mathrm{~cm}^{2}$ ).

\subsection{Design}

The test was designed through the three-factor mixed way, i.e. 2 (levels of processing: deep levels of processing-shallow levels of processing) $\times 4$ (types of pictures: misled-generate pictures-misled-read pictures-new pictures-old pictures) $\times 2$ (test conditions: warning-non-warning). Within variables were levels of processing and type of pictures, and between variables were test conditions. Dependent variables were response time and recognition accuracy.

\subsection{Procedures}

The subjects sat in front of a computer in a sound-proof electromagnetic shielding room, and got ready for test. Before the beginning of formal experiment, subjects should complete a practice experiment. At the practice phase, other procedures were the same as those at the experimental phase, but there was no misleading phase, so as to make subjects not guess the test objective for influencing the results of the formal experiment. The practice experiment mainly aimed to make subjects well know the basic contents of formal experiment and the contents of buttons corresponding to the tasks for every phase.

The experimental phase was composed of three phases. At the learning phase (i.e. the first stage), the figured literal story was presented to subjects sentence by sentence. According to the different processing tasks, the pictures were divided into two groups. In shallow levels of processing group, subjects were required to judge the background color of pictures (i.e. white or black). In deep levels of processing group, subjects were required to judge the valence of pictures (i.e. positive, neutral, or negative); and each subject was presented with the pictures from both groups in counterbalanced order. Procedures for each trial were as follows. One sentence of the story (a certain word or phrase of the sentence was highlighted) was first presented with an unlimited time in the middle of the computer screen, and disappeared when "Enter" was pressed after reading the screen. Then one 
cross-shaped fixation $(500 \mathrm{~ms})$ appeared in the middle of the computer screen, which was followed by the highlighted pictures $(1000 \mathrm{~ms})$. Subjects judged the background color or valence of the pictures within this time (1000 ms), with a subsequent buffer interval (1000 ms) (i.e. empty screen). Then subjects calculated for 3 minutes through the continuous-3-subtraction method in the answer card (i.e. a distraction task).

At the misleading phase (i.e. the second stage), the misleading literal summary of story was presented to subjects to be read aloud. In order to control the differences of difficulty, the difficulty was balanced among the misled-generate items, misled-read items and new items, which were presented in a counterbalanced way. Procedures for each trial were as follows. One sentence of the story (a certain word or phrase of the sentence was highlighted) was first presented with an unlimited time in the middle of the computer screen, and disappeared when "Enter" was pressed after reading the screen followed by a buffer interval (1000 ms) (i.e. empty screen). After the presentation of misleading literal summary, subjects calculated for 3 minutes through the continuous-3-subtraction method in the answer card (i.e. a distraction task).

After the ending of the misleading phase but before the beginning of the recognition phase, the warning variables were added. All subjects were divided into two groups: non-warning group and warning group. In the non-warning group, subjects were not warned about the authenticity of misleading literal summary and were presented with non-warning words before the retrieval; however, in the warning group, the subjects were warned that the misleading literal summary did not certainly consist with the contents of the read pictures, and were presented with warning words before the retrieval.

The recognition phase was the third stage. In order to minimize the arbitrariness of answer, subjects were told as follows before the beginning of recognition: if the subjects answered arbitrarily, the computer would automatically find such arbitrary answer, and subjects were required for retest. But in fact, no retest was made at all. At the recognition phase, subjects were required to judge whether four types of pictures had been presented (i.e. old/new judgment), including misled-generate pictures, misled-read pictures, new pictures, and old pictures. All pictures were randomly presented, and then subjects were required to further complete a Remember/Know/ Guess $(\mathrm{R} / \mathrm{K} / \mathrm{G})$ judgment (i.e. confidence judgment). Procedures for each trial were as follows. One crossshaped fixation $(500 \mathrm{~ms})$ appeared in the middle of the computer screen, which was randomly followed by one picture $(1000 \mathrm{~ms})$. Then the interface of $\mathrm{R} / \mathrm{K} / \mathrm{G}$ judgment appeared $(3000 \mathrm{~ms})$, with a subsequent buffer interval (1000 ms) (i.e. empty screen). The whole experiment lasted for 30 minutes on average. At the end of the test, all subjects were asked to guess the test objective, and then told of actual test objective.

\subsection{Data Management and Statistical Analysis}

The data were processed and analyzed through the SPSS16.0 statistical software. The data for tests with a response time of $>2000 \mathrm{~ms}$ or $<100 \mathrm{~ms}$ for old/new judgment were rejected. The main effect and interaction of levels of processing, type of pictures and warnings were analyzed mainly through the repeated measures variance analysis.

\section{Result}

\subsection{Accuracy of Old/New Judgment}

The three-factor repeated measures variance analysis was carried out. The descriptive statistics for types of pictures $\times$ test conditions $\times$ levels of processing interaction on accuracy of old/new judgment were shown in Table 1. The results showed that the main effect of test conditions was significant $(F(1,33)=13.20, p<.01)$, i.e. the recognition accuracy under the non-warning condition was significantly higher than that under the warning condition ( $M$ [non-warning-warning] $=0.13, p<.01)$. The main effect of type of pictures was also significant $(F(3,99)=12.89, p<.001)$. As indicated through post-hoc tests, the recognition accuracy of misled-generate pictures was significantly lower than that of old pictures $(M[$ misled-generate pictures-old pictures] $=-.15, p$ $<.01)$; the recognition accuracy of misled-read pictures was significantly lower than that of new pictures $(M$ [misled-read pictures-new pictures] $=-.05, p<.05)$ and old pictures $(M$ [misled-read pictures-old pictures] $=$ $-.19, p<.001)$; and the recognition accuracy of new pictures was significantly lower than that of old pictures $(M$ [new pictures-old pictures] $=-.14, p<.01$ ).

The interaction between test conditions and types of pictures was significant $(F(3,99)=13.57, p<.001)$. The simple effect was further analyzed as follows: 1) Under the different test conditions, the differences of recogni- 
Table 1. Means and SDs for types of pictures $\times$ test conditions $\times$ levels of processing interaction on accuracy of old $/$ new judgment.

\begin{tabular}{ccccc}
\hline & \multicolumn{2}{c}{ Non-warning condition } & \multicolumn{2}{c}{ Warning condition } \\
\cline { 2 - 5 } & Shallow processing & Deep processing & Shallow processing & Deep processing \\
\hline Misled-generate pictures & $.76(.21)$ & $.68(.24)$ & $.51(.34)$ & $.46(.33)$ \\
Misled-read pictures & $.75(.18)$ & $.55(.27)$ & $.50(.33)$ & $.42(.30)$ \\
New pictures & $.76(.24)$ & $.68(.25)$ & $.53(.35)$ & $.45(.35)$ \\
Old pictures & $.54(.27)$ & $.81(.24)$ & $.76(.24)$ & $.89(.16)$ \\
\hline
\end{tabular}

tion accuracies for different types of pictures (i.e. misled-generate pictures, misled-read pictures, new pictures, and old pictures) were significant $(F(1,33)=15.29, p<.001 ; F(1,33)=12.39, p<.01 ; F(1,33)=17.46, p<.001$; $F(1,33)=7.79, p<.01)$, i.e. the recognition accuracies of misled-generate pictures, misled-read pictures, and new pictures under the non-warning condition were significantly higher than those under the warning condition, but the recognition accuracy of old pictures under the non-warning condition was significantly lower than that under the warning condition; 2) Under the same test condition, the differences of recognition accuracies for different types of pictures were significant $(F(3,99)=21.08, p<.001)$, i.e. under the warning condition, the recognition accuracies of misled-generate pictures, misled-read pictures and new pictures were significantly lower than that of old pictures $(p<.001)$, but the differences of recognition accuracies were significant under the nonwarning condition $(F(3,99)=1.16, p>.05)$.

The interaction between types of pictures and levels of processing was significant $(F(3,99)=30.91, p<.001)$. The simple effect was further analyzed as follows. At deep levels of processing, the differences of recognition accuracies for different types of pictures were very significant $(F(3,99)=34.18, p<.001)$, i.e. at deep levels of processing, the recognition accuracy of old pictures was significantly higher than that of misled-generate pictures $(p<.001)$, misled-read pictures $(p<.001)$ and new pictures $(p<.001)$, and the recognition accuracies of misled-generate pictures and new pictures were significantly higher than that of misled-read pictures $(p<.01)$; but at shallow levels of processing, the differences of recognition accuracies for different types of pictures were not significant $(F(3,99)=0.17, p>.05)$.

The interaction of test conditions, types of pictures and levels of processing was significant $(F(3,99)=2.79, p$ $<.05)$. The simple effect was further analyzed as follows. Under the non-warning condition at shallow/deep levels of processing, the differences of recognition accuracies for different types of pictures were significant $(F(3,99)=9.17, p<.001 ; F(3,99)=6.72, p<.001)$, i.e. under the non-warning condition at shallow levels of processing, the recognition accuracy of old pictures was significantly lower than that of misled-generate pictures $(p<.01)$, misled-read pictures $(p<.01)$ and new pictures $(p<.01)$; and under the non-warning condition at deep levels of processing, the recognition accuracy of misled-read pictures was significantly lower than that of misled-generate pictures $(p<.01)$, new pictures $(p<.01)$ and old pictures $(p<.01)$. Moreover, under the warning condition at shallow/deep levels of processing, the differences of recognition accuracies for different types of pictures were significant $(F(3,99)=7.62, p<.001 ; F(3,99)=31.40, p<.001)$, i.e. under the warning condition at shallow/deep levels of processing, the recognition accuracy of old pictures was significantly higher than those of misled-generate pictures $(p<.01 ; p<.001)$, misled-read pictures $(p<.01 ; p<.001)$ and new pictures $(p$ $<.05 ; p<.001)$.

\subsection{Response Time of Old/New Judgment}

The descriptive statistics for types of pictures $\times$ test conditions $\times$ levels of processing interaction on response time of old/new judgment were shown in Table 2. The results showed that the main effect of test conditions was significant $(F(1,33)=28.51, p<.001)$, the recognition response time under the non-warning conditions was significantly longer than that under the warning conditions $(M$ [non-warning-warning] $=216.48, p<.001)$. The main effect of types of pictures was also significant $(F(3,99)=11.37, p<.001)$. As indicated through post-hoc tests, the recognition response time of misled-generate pictures was significantly longer than those of misledread pictures $(M$ [misled-generate pictures-misled-read pictures] $=-96.50, p<.001)$, new pictures $(M$ [misledgenerate pictures-new pictures] $=32.06, p<.05)$ and old pictures $(M$ [misled-generate pictures-old pictures $]=$ 
Table 2. Means and SDs for types of pictures $\times$ test conditions $\times$ levels of processing interaction on response time of old/ new judgment (ms).

\begin{tabular}{ccccc}
\hline & \multicolumn{2}{c}{ Non-warning condition } & \multicolumn{2}{c}{ Warning condition } \\
\cline { 2 - 5 } & Shallow processing & Deep processing & Shallow processing & Deep processing \\
\hline Misled-generate pictures & $1121.97(357.72)$ & $1092.49(341.41)$ & $951.51(309.06)$ & $915.73(302.14)$ \\
Misled-read pictures & $1113.06(287.64)$ & $950.6(333.96)$ & $900.35(270.28)$ & $731.66(248.85)$ \\
New pictures & $1071.49(361.34)$ & $1116.87(319.42)$ & $867.91(280.07)$ & $897.19(261.96)$ \\
Old pictures & $1085.58(364.97)$ & $1137.29(338.45)$ & $870.61(270.06)$ & $822.54(234.13)$ \\
\hline
\end{tabular}

$41.43, p<.05)$. The main effect of levels of processing was also significant $(F(1,33)=12.97, p<.01)$, i.e. the recognition response time at shallow levels of processing was significantly longer than that at deep levels of processing ( $M$ [shallow levels of processing-deep levels of processing] $=39.76, p<.01)$.

The interaction of between types of pictures and levels of processing was significant $(F(3,99)=11.06, p$ $<.001)$. The simple effect was further analyzed as follows. At deep levels of processing, the recognition response time showed significant effects of types of pictures $(F(3,99)=21.53, p<.001)$, i.e. the recognition response time of misled-generate pictures, misled-read pictures and old pictures was significantly longer than that of new pictures $(p<.001)$. However, at shallow levels of processing, the recognition response time did not show significant effects of types of pictures $(F(3,99)=2.65, p>.05)$.

\subsection{R/K/G Judgment}

The results for types of pictures $\times$ test conditions $\times$ levels of processing interaction on $R / K / G$ judgment (i.e. confidence judgment) after the inaccurate recognition were shown in Table 3. Under either warning or nonwarning condition, after misled-generate pictures, misled-read pictures and new pictures were judged as old, a $\mathrm{R}$ judgment was made at a far more amount than the sum of $\mathrm{K}$ judgment and $\mathrm{G}$ judgment, i.e. after the inaccurate recognition of misled-generate pictures, misled-read pictures and new pictures, the subjects were very convinced to clearly remember but not know or guess that the picture had appeared at the learning phase. However, after the old pictures were judged as new, a $\mathrm{R}$ judgment was made at a nearly equivalent amount to the sum of $\mathrm{K}$ judgment and $\mathrm{G}$ judgment, i.e. compared with other three types of pictures, after the inaccurate recognition of old pictures, the subjects were convinced less to remember but more to guess that the picture had not appeared, which increased the probability of $\mathrm{K}$ judgment or G judgment.

The retrieval of memory suggestibility for misled-generate pictures and misled-read pictures was estimated through the following processing estimation formula of $\mathrm{R} / \mathrm{K} / \mathrm{G}$ paradigm: $\mathrm{R}$ (intentional processing) $=\mathrm{P}$ (probability of remembering); A (automatic processing) = P (probability of knowing)/(1 - R) (Plancher et al., 2008). The results were shown in Table 4. Whatever levels of processing and test conditions, intentional processing was far more than automatic processing during the generation of memory suggestibility for misled-generate pictures and misled-read pictures. In addition, compared with that under the non-warning condition, intentional processing appeared at a higher probability (i.e. nearly 1.00) during the generation of memory suggestibility for misled-generate pictures and misled-read pictures under the warning condition.

\section{Discussion}

In our study, the recognition accuracies of misleading pictures was significantly lower than those of non-misleading pictures (i.e. old pictures and new pictures), indicating that it was valid to set the misinformation at the misleading phase. From Lindsay et al. (2004), the actual memories were distorted by the hinting, so that the individuals recalled the details or events they did not ever experience. As inferred by the author, after the receipt of misinformation, the new information would be integrated into original memory trace, which might be re-encoded to distort the memories during the recall or recognition, and moreover, such phenomenon might be inherent and its influences could generally not be perceived.

The interaction between types of pictures and levels of processing was significant, which demonstrated that levels of processing at the encoding phase were one of the important influencing factors for memory suggestibility. The recognition accuracies of old pictures was significantly higher than those of misled-generate pictures, 
Table 3. The probabilities of $\mathrm{R} / \mathrm{K} / \mathrm{G}$ judgments for different types of pictures after the inaccurate recognition.

\begin{tabular}{ccccc}
\hline & \multicolumn{2}{c}{ Non-warning condition } & \multicolumn{2}{c}{ Warning condition } \\
\cline { 2 - 5 } & Shallow processing & Deep processing & Shallow processing & Deep processing \\
\hline Misled-generate pictures & $.87 / .04 / .09$ & $.91 / .02 / .07$ & $.97 / .01 / .02$ & $.95 / .03 / .02$ \\
Misled-read pictures & $.77 / .09 / .14$ & $.78 / .07 / .15$ & $.95 / .02 / .03$ & $.96 / .02 / .02$ \\
New pictures & $.77 / .12 / .11$ & $.83 / .07 / .10$ & $.93 / .05 / .02$ & $.98 / .00 / .02$ \\
Old pictures & $.59 / .24 / .17$ & $.56 / .26 / .18$ & $.55 / .23 / .22$ & $.67 / .30 / .03$ \\
\hline
\end{tabular}

Note: R: Remember; K: Know; G: Guess.

Table 4. The probabilities of automatic processing and intentional processing of inaccurate retrieval for misleading pictures.

\begin{tabular}{ccccc}
\hline & \multicolumn{2}{c}{ Non-warning condition } & \multicolumn{2}{c}{ Warning condition } \\
\cline { 2 - 5 } & Shallow processing (R/A) & Deep processing (R/A) & Shallow processing (R/A) & Deep processing (R/A) \\
\hline Misled-generate pictures & $.87 / .31$ & $.91 / .22$ & $.97 / .33$ & $.95 / .60$ \\
Misled-read pictures & $.77 / .39$ & $.78 / .32$ & $.95 / .40$ & $.96 / .50$ \\
Total & $.82 / .35$ & $.85 / .27$ & $.96 / .37$ & $.96 / .55$ \\
\hline
\end{tabular}

Note: R: Intentional processing; A: Automatic processing.

misled-read pictures and new pictures at deep levels of processing, i.e. memory suggestibility was greater at deep levels of processing, and intentional processing at the encoding phase was more beneficial to the generation of memory suggestibility. As shown by earlier study, compared with shallow levels of processing, deep levels of processing increased the incidence rates of accurate and false memories, which might be influenced by the characteristics of materials and the directions of processing (Chan, McDermott, Watson, \& Gallo, 2005). In our study, the recognition response time of pictures at shallow levels of processing was significantly longer than that of pictures at deep levels of processing. Therefore, compared with automatic processing at the encoding phase, if the prototypes of misleading pictures (i.e. original pictures) were consciously processed at the encoding phase, the recognition of misleading pictures became faster, which indicated intentional processing at the encoding phase increased the fluency of retrieval at the recognition phase (Thomas, Bulevich, \& Chan, 2010).

The interaction between types of pictures and test conditions was significant, which illustrated that the warnings was certainly one of the important influencing factors for memory suggestibility, consisting with the conclusions of existing studies (Thomas, Bulevich, \& Chan, 2010). Compared with those under the warning condition, the recognition accuracies of misled-generate pictures and misled-read pictures were significantly higher, but the recognition accuracy of old pictures was significantly lower under the non-warning condition. Therefore, warnings at the retrieval phase increased memory suggestibility, which conflicted with the mainstream viewpoints that warnings reduced the false memories, possibly attributing to the different stages where the warning words emerged. Gallo, Roberts, \& Seamon (1997) examined how the associative false memories were influenced by the types and emergent stages of warning words, in which subjects were divided into three groups: Group 1 (presenting standard instructions of DRM paradigm), Group 2 (presenting warnings before the learning) and Group 3 (presenting warnings after the learning but before the test). The result showed that warnings did not influence false memories in Group 3, but effectively reduced the inaccurate recognition in Group 2, and false memories was produced at a higher probability at the learning phase than that at the test phase. Since the warnings were set before the test phase in present research, subjects bore a certain psychological stress to make a more intentional monitoring processing (i.e. intentional processing) at the test phase, which confused the original information with misinformation to increase memory suggestibility for misleading pictures. As shown by the relevant studies on the effects of intentional processing on false memories, the intentional processing not only could not help the individuals refuse the lure items, but also might actually damage their abilities of reducing false memories (Hicks \& Marsh, 2001). In a word, the intentional processing caused by warnings during the retrieval not only could not make subjects effectively refuse the misleading items, but also reduced their abilities 
of identifying the interferential information to increase memory suggestibility. Moreover, the warnings increased the memory accuracy for non-misleading pictures, indicating that the warnings made subjects more recall the non-misinformation to increase the response accuracy.

In addition, the interaction between test conditions and levels of processing was significant on the generation of memory suggestibility. Under the warning condition at shallow/deep levels of processing, the recognition accuracy of old pictures was significantly higher than that of misled-generate pictures, misled-read pictures and new pictures, which demonstrated whatever levels of processing of original information at the encoding phase, the warnings emerging before the memories retrieval made subjects pay more attention to information viewed or remembered in the past. The more attention would easily confuse the original information with misinformation, thus causing a greater memory suggestibility. However, under the non-warning condition at shallow levels of processing, the recognition accuracy of old pictures was significantly lower than that of misleading pictures and new pictures. Thus, we speculated that the unconscious processing of pictures at the encoding phase might blur the memories of details of original pictures; accordingly, the recognition for old pictures would become uncertain due to the weak memories trace, so as to cause a higher randomness and guess to increase the inaccurate recognition.

The response time of misled-generate pictures was significantly longer than that of other three pictures (especially the misled-read pictures), i.e. the highly-intentional processing of misled-generate items at the misleading phase would confuse more misinformation with the original information, and more recall was required with a greater difficulty in judgment for recognition of misled-generate pictures than that for other three pictures. As shown by the existing studies, compared with misled-read items, misled-generate items produced a higher probability of recall, thus naturally causing a longer response time (Holliday \& Hayes, 2001). In addition, at deep levels of processing, the effects of types of pictures were significant on response time, because the prototypes of misled-generate pictures, misled-read pictures and old pictures were processed intentionally at the encoding phase, yet new pictures were not involved at the encoding phase so as to recall faster without the interference of original information.

As another important result, whatever levels of processing and test conditions, a less confidence was produced at the inaccurate recognition for old pictures than that for the misled-read pictures, misled-generate pictures and new pictures, which had been well known. Loftus et al. (1989) indicated that subjects in the misleading group retrieved faster and became more confident for the inaccurate recognition through the research of response time and confidence levels by self-report. Zaragoza \& Mitchell (1996) found that a high confidence was often accompanied by the inaccurate report of misleading details. The generation of memory suggestibility was accompanied by a high level of confidence, because the original information was seriously confused with misinformation by intentional processing at the encoding and misleading phase, eventually leading to an inaccurate judgment depending on recollection at the retrieval phase, which showed high confidence.

Whatever levels of processing and test conditions, intentional processing was far more than automatic processing during the generation of memory suggestibility for misled-generate pictures and misled-read pictures. Therefore, the generation of memory suggestibility was more attributed to intentional processing. According to the demands characteristics hypothesis of McCloskey \& Zaragoza (1985), the misled subjects might remember not only the original information, but also the afterward misinformation. Nevertheless, the misinformation was chosen mainly due to the inductive effects of external demands on subjects' reports for the misleading items, possibly indicating that intentional processing contributed greatly to the effects of misinformation. From Holliday \& Hayes (2000), as assessed through the process dissociation procedure (PDP), both automatic processing and intentional processing played a role in children's receipt of misinformation, but the effects of automatic processing would decrease with the age. In this study, the adult subjects (i.e. university students) had a mature cognitive ability, and thus had the contribution of automatic processing been minimized in these subjects? Or had such effect been reduced to the critical value at an earlier time? In future research, age effects of the contributions of intentional and automatic processing on memory suggestibility should be further explored.

Compared with that under the non-warning condition, intentional processing appeared at a higher probability (i.e. nearly 1.00) in memory suggestibility for misleading pictures under the warning condition, which indicated that warnings at the retrieval phase increased the probability of intentional retrievals during the generation of memory suggestibility. If the misinformation was received mainly through automatic processing, subjects were warned that the overlooks of misinformation might hardly influence the memories of witnessed event (Lindsay et al., 2004). In the current study, the warning was of a significant influence, which illustrated again that misin- 
formation was received mainly through intentional processing.

The generation of memory suggestibility not only was the result of interaction among the encoding, misleading and retrieval, but also was the result of interaction between automatic processing (activation) and intentional processing (monitoring). The contributions of automatic and intentional processing are not decided by some factor, which should be determined by various factors during the encoding, misleading and retrieval. The automatic and intentional processing is likely to be the main reason for inducing memory suggestibility. The activation/monitoring dual-processing theory might be applied to explain the mechanism of memory suggestibility.

Nevertheless, this study has explored the mechanism of memory suggestibility preliminarily, which belongs to the exploratory research. The conclusions of this research should be proved through more similar researches. At the same time, the current research has just taken a small step in the integrated domain, and further research is needed to integrate the important influencing factors at the encoding, misleading and retrieval phase for exploring the effects of them on memory suggestibility, construct rational models, and standardize the research materials.

\section{Acknowledgements}

The authors wish to thank Ke Du and Xiao-Xia Yang for helpful suggestions. We also express our appreciation to all the investigators for assistance with data collection and coding.

\section{References}

Arndt, J. (2006). Distinctive Information and False Recognition: The Contribution of Encoding and Retrieval Factors. Journal of Memory and Language, 54, 113-130. http://dx.doi.org/10.1016/j.jml.2005.08.003

Brainerd, C. J., \& Reyna, V. F. (1998). Fuzzy-Trace Theory and Children's False Memories. Journal of Experimental Child Psychology, 71, 81-129. http://dx.doi.org/10.1006/jecp.1998.2464

Chan, J. C. K., McDermott, K. B., Watson, J. M., \& Gallo, D. A. (2005). The Importance of Material-Processing Interactions in Inducing False Memories. Memory \& Cognition, 33, 389-395. http://dx.doi.org/10.3758/BF03193057

Dewhurst, S. A., Bould, E., Knott, L. M., \& Thorley, C. (2009). The Roles of Encoding and Retrieval Processes in Associative and Categorical Memory Illusions. Journal of Memory and Language, 60, 154-164. http://dx.doi.org/10.1016/i.jml.2008.09.002

Gallo, D. A., Roberts, M. J., \& Seamon, J. G. (1997). Remembering Words Not Presented in Lists: Can We Avoid Creating False Memories? Psychonomic Bulletin \& Review, 4, 271-276. http://dx.doi.org/10.3758/BF03209405

Gallo, D. A., Roediger, H. L., \& McDermott, K. B. (2001). Associative False Recognition Occurs without Strategic Criterion Shifts. Psychonomic Bulletin \& Review, 8, 579-586. http://dx.doi.org/10.3758/BF03196194

Guo, X. Y., Wan, L. L., Guo, X. R., \& Wei, Z. C. (2005). A Preliminary Exploration into the Unconsciousness Mechanism in False Memories. Psychological Science, 28, 362-367.

Hicks, J. L., \& Marsh, R. (2001). False Recognition Occurs More Frequently during Source Identification than during OldNew Recognition. Journal of Experimental Psychology: Learning, Memory, and Cognition, 27, 375-383. http://dx.doi.org/10.1037/0278-7393.27.2.375

Holliday, R. E., \& Hayes, B. K. (2000). Dissociating Automatic and Intentional Processes in Children's Eyewitness Memory. Journal of Experimental Child Psychology, 75, 1-42. http://dx.doi.org/10.1006/jecp.1999.2521

Holliday, R. E., \& Hayes, B. K. (2001). Automatic and Intentional Processes in Children's Eyewitness Suggestibility. Cognitive Development, 16, 617-636. http://dx.doi.org/10.1016/S0885-2014(01)00042-9

Li, H. Y., Lian, R., \& Weng, J. (2008). Effects of Forewarning, Repetition and Feedback on False Memories: An Analysis with the Signal Detection Theory. Psychological Science, 31, 1507-1509.

Lindsay, S., Hagen, L., Read, J. D., Wade, K., \& Garry, M. (2004). True Photographs and False Memories. Psychological Science, 15, 149-154. http://dx.doi.org/10.1111/j.0956-7976.2004.01503002.x

Loftus, E. F., Donders, K., Hoffman, H. G., \& Schooler, J. W. (1989). Creating New Memories That Are Quickly Accessed and Confidently Held. Memory \& Cognition, 17, 606-617. http://dx.doi.org/10.3758/BF03197083

McCloskey, M., \& Zaragoza, M. (1985). Misleading Postevent Information and Memory for Events: Arguments and Evidence for Memory Impairment Hypotheses. Journal of Experimental Psychology: General, 114, 1-16. http://dx.doi.org/10.1037/0096-3445.114.1.1

McDermott, K. B., \& Watson, J. M. (2001). The Rise and Fall of False Recall: The Impact of Presentation of Associates. Journal of Memory and Language, 45, 160-176. http://dx.doi.org/10.1006/jmla.2000.2771 
Peters, M. J. V., Jelicic, M., Gorski, B., Sijstermans, K., Giesbrecht, T., \& Merckelbach, H. (2008). The Corrective Effects of Warning on False Memories in the DRM Paradigm Are Limited to Full Attention Conditions. Acta Psychologica, 129, 308-314. http://dx.doi.org/10.1016/j.actpsy.2008.08.007

Plancher, G., Nicolas, S., \& Piolino, P. (2008). Influence of Suggestion in the DRM Paradigm: What State of Consciousness Is Associated with False Memories? Consciousness and Cognition, 17, 1114-1122. http://dx.doi.org/10.1016/i.concog.2008.08.006

Rhodes, M. G., \& Anastasi, J. S. (2000). The Effects of a Level-of-Processing Manipulation on False Recall. Psychonomic Bulletin \& Review, 1, 158-162. http://dx.doi.org/10.3758/BF03210735

Thomas, A. K., Bulevich, J. B., \& Chan, J. C. K. (2010). Testing Promotes Eyewitness Accuracy with a Warning: Implications for Retrieval Enhanced Suggestibility. Journal of Memory and Language, 63, 149-157.

http://dx.doi.org/10.1016/j.jml.2010.04.004

Tussing, A., \& Greene, R. (1997). False Recognition of Associates: How Robust Is the Effect? Psychonomic Bulletin \& Review, 4, 572-576. http://dx.doi.org/10.3758/BF03214351

Westerberg, C. E., \& Marsler, C. J. (2006). Do Instructional Warnings Reduce False Recognition? Applied Cognitive Psychology, 20, 97-114. http://dx.doi.org/10.1002/acp.1170

Yang, Z. L., Wang, S. R., \& Tang, J. H. (2006). When Do False Memories Happen: Encoding/Maintaining Phase? Chinese Journal of Applied Psychology, 12, 99-106.

Ye, M. L., \& Liu, X. L. (2008). A Study on the Effects of Level Processing on False Memories. Psychological Research, 1, 67-70.

Zaragoza, M. S., \& Mitchell, K. J. (1996). Repeated Exposure to Suggestion and the Creation of False Memories. Psychological Science, 7, 294-300. http://dx.doi.org/10.1111/j.1467-9280.1996.tb00377.x

Zhou, C., \& Nie, J. (2009). An Empirical Research on the Dual-Processing Mechanism of False Recognition. Psychological Science, 32, 334-337.

Zhou, L. H., \& Liu, A. L. (2003). The Summarize of Research on Suggestibility of Children's Memory. Advances in Psychological Science, 11, 534-540. 